\title{
Les chasseurs komis et le droit coutumier dans le contexte de l'animisme
}

Komi jahimeeste arusaam tavaõigusest animismi taustal

\section{Art Leete et Lipin Vladimir}

Traducteur : Eva Toulouze

\section{OpenEdition \\ Journals}

Édition électronique

URL : https://journals.openedition.org/efo/7526

DOI : 10.4000/efo.7526

ISSN : 2275-1947

Éditeur

INALCO

\section{Référence électronique}

Art Leete et Lipin Vladimir, "Les chasseurs komis et le droit coutumier dans le contexte de

l'animisme », Études finno-ougriennes [En ligne], 49-50 | 2018, mis en ligne le 14 janvier 2019, consulté le 06 octobre 2022. URL : http://journals.openedition.org/efo/7526 ; DOI : https://doi.org/10.4000/efo. 7526

Ce document a été généré automatiquement le 6 octobre 2022.

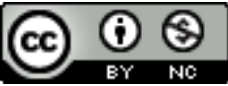

Creative Commons - Attribution - Pas d'Utilisation Commerciale 4.0 International - CC BY-NC 4.0

https://creativecommons.org/licenses/by-nc/4.0/ 


\title{
Les chasseurs komis et le droit coutumier dans le contexte de l'animisme
}

\author{
Komi jahimeeste arusaam tavaõigusest animismi taustal
}

\author{
Art Leete et Lipin Vladimir
}

Traduction : Eva Toulouze

1 L'étude du droit coutumier des chasseurs permet de comprendre la vision animiste du monde $^{1}$. Dans le domaine de la chasse, les perceptions archaïques du monde ont survécu plus longtemps que dans d'autres sphères où les effets de la modernisation se sont diffusés plus facilement. Le lien signifiant entre les règles régissant les pratiques cynégétiques et les croyances fonctionne dans la communauté tant que ces deux dimensions (le mode de vie des chasseurs et la perception animiste du monde) demeurent suffisamment d'actualité. Or ceci veut dire que les chasseurs sont conscients en même temps des règles du droit coutumier ainsi que des principes de l'animisme, et qu'ils reconnaissent les unes comme les autres, le droit coutumier de manière assurée et l'animisme d'une manière plus ou moins précise.

2 Les premiers anthropologues voyaient dans le droit coutumier un mécanisme de contrôle social au sens le plus large, depuis les règles de politesse jusqu'aux mesures punitives (Fuller, 1994). Bronislaw Malinowski (1926, p. 2) définit le droit coutumier comme un ensemble de forces qui produisent dans «la tribu sauvage» ordre, unité et harmonie. Chez les peuples premiers, le droit couvre toute la vie quotidienne et les activités publiques, depuis les plus insignifiantes jusqu'aux plus évidemment importantes.

3 La question des rapports entre le droit et les coutumes au sens le plus général n'a pas cessé d'occuper le devant de la scène dans l'anthropologie juridique (WIPO, 2013, p. 2, 12). Ce qui a posé problème a été l'application inconditionnelle des catégories juridiques occidentales pour comprendre le droit des sociétés des peuples premiers. À la fin $\mathrm{du} \mathrm{xx}^{\mathrm{e}}$ siècle, l'éventualité, même la plus indéterminée, de distinguer le droit 
coutumier de manière claire a constitué, dans le traitement du droit coutumier des peuples premiers, un défi sui generis: car les systèmes juridiques des États avaient intégré toutes les communautés et les individus dans la sphère du droit officiel (Fuller, 1994).

La question fondamentale est ainsi celle d'identifier des traits distinctifs, qui pourraient distinguer le droit coutumier de communautés données des systèmes juridiques plus largement répandus. Les relations entre le droit, la vie sociale et l'environnement naturel vont dans les deux sens et tendent à préserver l'équilibre, mais il faut parvenir à faire ressortir la traits identificateurs qui servent de lien entre les membres de la communauté (WIPO, 2013, p. 2, 6, 12, 14). Par exemple, Émile Durkheim voit dans le droit un symbole extérieur et visible de la solidarité sociale. Bien que le droit ne relève pas de la sphère matérielle ( $d$ 'autant que pour ce qui est du droit coutumier, il ne comporte pas de forme écrite), il ne manque pas d'avoir des effets sensibles. Le caractère du droit détermine la forme et la mesure que prend le lien entre les personnes, de même qu'il peut découler de ces mêmes relations (Durkheim, 1984 [1893], p. 24-25; voir aussi Geertz, 1983, p. 232 ; Fuller, 1994).

5 Les coutumes sont le fondement du droit et le droit coutumier, qui ne prétend pas dépasser les bornes de la communauté, est un phénomène culturellement unique (Svensson, 2002, p. 4-5). Le droit coutumier est la fusion de toute la vie, les valeurs, la vision du monde et l'auto-identification des personnes (WIPO, 2013, p.6, 10, 18). Clifford Geertz (1983, p. 184) voit dans le droit un système de significations culturelles, une manière originale de sonner du sens et en partie de créer explicitement un monde. Le droit est pour lui un outil conceptuel pour représenter et façonner la réalité sociale (idem, p. 234).

6 Déjà Malinowski $(1926$, p. 10) critiquait la tendance des chercheurs de son temps à expédier le droit coutumier des peuples premiers par la peur du «châtiment surnaturel ». Il n'est cependant pas forcément erroné de supposer que la perception animiste du monde confère au droit coutumier par exemple des chasseurs une spécificité qui ne lui permet pas de se fondre avec le discours juridique officiel. Les connaissances sur le droit coutumier et sur ses applications réelles sont d'autant plus faciles à suivre qu'elles font partie explicitement de la pratique religieuse. Les croyances animistes servent de fondement idéologique, de source nourricière au droit coutumier et sa pratique reflète la vision du monde, elle y est entrelacée, même si les personnes concernées peuvent, la plupart du temps, ne pas en parler explicitement.

7 Dans les récits des explorateurs du XIX ${ }^{\mathrm{e}}$ siècle (voir par exemple Latkin, 1853 ; Popov, 1974 ; Abramov, 1914) et dans les études des ethnographies professionnels (Žakov, 1990 [1901] ; Nalimov, 2010 [1924] ; Sidorov, 1997 [1928] ; Konakov, 1983 ; Il'ina, Ul'ašev, 2009), la perspective historique et le système du droit coutumier des chasseurs komis est bien documenté et étudié. L'image mentale des hommes komis qui errent de par la forêt bleue sombre, est devenue l'un des prototypes non seulement artistique, mais aussi scientifique de la culture komie. Les études les plus anciennes sur la culture komie sont pleines de descriptions d'hommes de la forêt presque feériques. Comme on pourrait s'y attendre, tous les anciens classiques de l'ethnographie komie ont travaillé sur la chasse. Nous aussi, nous avons déjà consacré un article à la présentation des principes généraux du droit coutumier komi ainsi qu'à celle de nos données de terrain (Lipin, Leete, 2000). De même, nous avons consacré deux ou trois études plus anciennes 
aux points de contact entre le contexte animiste et le droit coutumier (Leete, Lipin, 2012 ; 2015 ; Lipin, Leete, 2015).

8 Avec cet article, nous avançons dans l'étude du droit coutumier komi et nous analysons la pratique contemporaine de la chasse dans le cadre de l'interprétation et de l'application du droit coutumier. Il n'est pas particulièrement compliqué de formuler le rapport entre le droit coutumier et la vision du monde animiste. Les chasseurs komis partent du principe qu'il faut être honnête et dire la vérité, car l'imprécision qui dérive d'une formulation vague crée un état d'esprit qui permet l'actualisation de la vision du monde animiste. Mais comment partir de ce principe dans la vie réelle? Pour le savoir, il suffit de suivre les actes et les propos des chasseurs et les comparer aux données antérieurement publiées par les chercheurs ainsi que leurs interprétations.

Notre article ne prétend pas donner un aperçu systématique de tout ce qui se rapporte au droit coutumier des chasseurs komis. Nous suivons une approche inductive : nous partons de nos observations et matériaux de terrain, ainsi que des conversations que nous poursuivons depuis des années. Nous avons ensuite ajouté à notre analyse une couche d'ethnographie historique que la base de la littérature spécialisée, en partant des auteurs qui avaient émergé au cours de nos conversations. Plus tard, nous avons abordé les tentatives de généralisation et les débats théoriques. Néanmoins, notre approche est fragmentaire, car nous n'avons pas cherché à collecter des données spécifiques à l'étude de l'éthique de la chasse et du droit coutumier. C'est là un thème qui, au milieu d'autres questions, est toujours revenu dans nos débats à différentes reprises. Nous estimons que les généralisations issues des matériaux ethnographiques anciens sont adéquates et qu'il n'est pas nécessaire d'en donner une fois de plus un aperçu structurel. Nous voulons faire ressortir les thèmes que les chasseurs komis eux-mêmes ont fait ressortir, sans que nous ne les ayons aucunement orientés.

\section{Lois de l'État et lois de la forêt}

10 Les chasseurs komis parlent de leur droit coutumier comme d'un droit qui s'oppose aux lois ou qui en est indépendant. Pourtant, il a existé entre les deux une longue relation particulièrement délicate. D'une certaine manière et dans une certaine mesure le droit coutumier des Komis a été au cours des siècles encadré avec souplesse par les textes juridiques centraux de l'État russe. Ainsi, Ivan le Terrible, au Xvi siècle, a conféré par un document écrit, une gramota, le droit aux habitants de la Grande Bjarmija, c'est-à-dire des terres komies, le droit au "profit sylvestre » (c'est-à-dire le droit de chasser) toute l'année et sans limitations officielles (Bunakov, 2010 [1857], p. 32). Vers la fin du Xvi ${ }^{e}$ siècle, la répartition des zones de chasse des Komis a été officiellement fixée village par village (Istorija Komi, 2011, p. 91-92). Telle était la politique générale de la Russie tsariste dans les zones des «terres noires ", là où le servage n'existait pas, où la terre appartenait à l'État, mais était confiée aux communautés paysannes qui en tiraient leur usufruit (Konakov, 1983, p. 166). Ainsi, le droit de chasser et la répartition des territoires de chasse n'a pas été de tous temps seulement réglementé par voie orale. Les droits à la chasse des Komis ont été ainsi inscrits également dans des documents écrits, c'est-à-dire qu'ils ont été réglementés par la législation. Certes, avec sa gramota, Ivan le Terrible ne faisait que confirmer une pratique coutumière ancestrale, mais par cet acte la chasse chez les Komis est devenue un domaine réglementé par le droit écrit, inscrit dans la législation étatique. Il est vrai que cette réglementation restait en fait 
nominale, car elle fixait également le principe que l'État ne se mêlait pas des relations entre chasseurs. Il n'en reste pas moins que dès la fin du XvI ${ }^{\text {esiècle }}$ Nénetses et Ougriens de l'Ob ont présenté de nombreuses plaintes contre des Komis et des Russes pour infraction au droit sur les territoires de chasse (Istorija Komi, 2011, p. 92). Ainsi, les peuples premiers de la région, il y a déjà des siècles, avaient besoin de l'intervention de l'État pour réglementer l'utilisation des territoires de chasse. En même temps, la conception du droit coutumier des chasseurs komis dans une période plus proche d'aujourd'hui a été plutôt dominée par le principe de la souveraineté juridique des chasseurs, et elle a reposé sur la tentative d'éviter toute interférence étatique dans les affaires de chasse.

11 Comme l'affirme Vassili Nalimov (2010 [1924], p. 81 ; voir aussi Il'ina, Ul'ašev, 2009, p.115), le droit coutumier komi repose sur la confiance et le respect mutuel des chasseurs. Nalimov souligne également que les lois de l'État ne s'appliquent pas dans la forêt komie, car il n'y a personne pour en suivre l'application et pour la garantir. En 1908, il écrit plus dans le détail, et présente quelques détails concrets des principes régissant les coutumes komies. Il présente des extraits de documents qui prouvent que les Komis, au niveau local, pour résoudre les conflits judiciaires, partaient toujours des coutumes populaires et ne s'exprimaient que lorsque les textes officiels entraient en conflit avec le droit coutumier, précisant qu'ils n'avaient pas l'intention de respecter la loi. D'après Nalimov, les Komis étaient convaincus qu'il faudrait élaborer des lois komies ad hoc, basées sur «les coutumes locales et les spécificités ethniques de la région » (Survo, Šarapov, 2015, p. 166-167).

Même si l'État n'a pas suivi les demandes des Komis et ne leur a pas permis d'avoir leurs lois à eux, les chasseurs continuent à suivre les coutumes locales. Le caractère des normes reconnues par les communautés était déterminé par l'isolement social et psychologique lors des expéditions de chasse et par des considérations économiques. L'objectif des règles de chasse est de prévenir les conflits ou d'en proposer une solution pacifique. Parmi les valeurs centrales dans le droit de la forêt komie, relevons l'abord l'honnêteté, mais aussi la prise en compte des intérêts des uns des autres et la place prioritaire accordée aux intérêts collectifs (Konakov, 1983, p. 176-178).

D'après Konakov (idem: p.178-179) les relations avec la nature, elles aussi, les conceptions animistes, font partie des «normes juridiques populaires». Le droit coutumier komi a pour stratégie un rapport économe aux ressources naturelles, déterminé par le contexte particulier de leur culture spirituelle.

Il est imprégné de représentations animistes. Par exemple l'un des principes essentiels de l'animisme ainsi que du droit coutumier et de l'éthique de la chasse est qu'on n'a pas le droit de parler de manière explicite des résultats attendus de la chasse et du gibier. Abramov (1914, p. 90) raconte un cas qu'il a vécu : un membre d'un artel de chasse komi a prédit à l'automne une bonne chasse hivernale, ayant constaté à l'automne une grande quantité d'écureuils. un chasseur plus expérimenté l'a mis en garde, et lui a dit qu'il ne fallait pas à l'avance vanter la chasse, car les écureuils pouvaient simplement être de passage. Cette interdiction de parler explicitement d'une bonne chasse et de manière générale de tout ce qui concerne la chasse a été largement documentée chez les Komis (voir par exemple Sidorov, 1997 [1928], p. 146 ; Konakov, 1983, p. 191-193 ; Il'ina, Uljašev, 2009, p. 105, 111-112). L'interdiction de la parole explicite s'articule avec la croyance des Komis en la puissance magique du lien entre la parole et les événements de la vie humaine. 
15 La magie chez les chasseurs a ses traits spécifiques, comparée avec celle qui règne au village, car elle se concentre sur l'explication de la malchance (Konakov, 1983, p. 197-198) et elle pénètre toutes les paroles et les actes des chasseurs. La forme de sorcellerie la plus directement liée à la chasse porte chez les Komis le nom de vomidz: il s'agit d'un dommage magique provoqué par une parole délibérée ou fortuite exprimant la jalousie, la force, la santé, le succès ou la réussite pour la personne ou l'objet dont il est question (Sidorov, 1997 [1928], p.139-140, 147, 167-168); mais le vomidz peut émerger tout simplement avec la présence d'une personne inconnue ou à l'aide de pensées négatives (idem, p. 45-146). Quand un chasseur est poursuivi par la malchance, ce fait est toujours mis en rapport avec des circonstances référant à la magie. Par exemple, si on regarde plus loin dans l'histoire de la sorcellerie komie, encore au début du siècle dernier les chasseurs pensaient que les pièges où un inconnu a volé la prise, perdent leur pouvoir d'attraction et ont été endommagés magiquement après le vol (Sidorov, 1997 [1928], p. 86).

16 Dans le cas de la chasse, les Komis relèvent encore une forme spécifique de vomidz, appelée urknittsöm. Le chasseur doit être serein, car le déséquilibre spirituel, l'expression incontrôlée de sentiments, le rapport superficiel au gibier débouche sur la malchance, le gibier peut être endommagé (par exemple la peau de l'animal se déchire, ou bien le chasseur ne trouve pas l'oiseau qu'il a tiré, qui disparaît dans les fourrés ou est emporté par le courant). L'urknittsöm peut aussi déboucher sur l'«endommagement » du chasseur lui-même, qui perd son aptitude à tirer ou à s'orienter, perd la tête ou commence à avoir des visions (idem, p. 170-173). L'acte de sorcellerie peut priver également le chien du chasseur de ses qualités utiles (odorat, insistance à poursuivre le gibier (Konakov, 1983, p. 197). Les résultats du vomidz peuvent susciter des tensions entre les chasseurs partis en expédition, ce qui met en cause la réussite générale de la chasse, comme le souligne Kallistrat Žakov (1990 [1901], p. 323-324).

17 Le rapport aux propos sur la chasse et au comportement des chasseurs reflète à première vue le rapport ambivalent entre l'honnêteté (dire la vérité), entre la sincérité des attitudes et une mystérieuse réserve. Pour éviter le vomidz, la différence ou l'ambivalence entre propos et vérité sont d'emblée codifiées dans la stratégie narrative des chasseurs. Ceux-ci approuvent les propos dont on ne peut pas savoir s'ils sont vrais ou faux. une véritable histoire de chasse est ainsi en même temps vérité et mensonge, et on essaye de cacher à l'auditeur le véritable statut de tel ou tel propos : le mensonge doit apparaître comme véridique, la vérité comme mensongère. À l'écoute d'une telle histoire, l'hésitation est totale, mais c'est là en même temps l'un des mécanismes d'activisation de la vision du monde animiste. Les chasseurs komis parlent de tout ce qui a rapport à la forêt par un décalage narratif, qui permet de préserver le potentiel magique de la chasse (voir Leete, Lipin, 2012, p. 295-298 ; 2015, p. 81).

18 Dans ce contexte, on comprend bien la différence de principe entre les fondements et les sources du droit officiel et du droit coutumier. L'indétermination magique du droit coutumier des chasseurs komis et l'aspiration à la précision du droit écrit ne s'harmonisent aucunement, et pourtant il existe entre eux un certain lien qu'on pourrait sous condition appeler un rapport amour-haine. Aujourd'hui par exemple, les chasseurs komis affirment ne vouloir aucun lien avec les autorités locales, avec les organes de surveillance ou de maintien de l'ordre, et en même temps ils se plaignent si l'administration du raïon ne délivre à personne le permis de chasse (TT, 2013). Mais si 
les autorités laissent une grande partie de la forêt aux chasseurs pour qu'ils en usent selon leur bon vouloir, on sera content de leur comportement, estimé adéquat. En Russie, ce n'est pas une habitude de demander des permis de chasse, mais les chasseurs komis voient leurs droits autonomes comme fondamentaux, hérités de leurs ancêtres. La diversité encore existante et l'efficacite des règles coutumières confirment que les chasseurs komis sont capables d'utiliser leurs privilèges traditionnels de manière pertinente.

\section{Une ethnographie de la loi sur la forêt}

Bien que traditionnellement les lois de la forêt soient par nature magiques, une grande partie, toutefois, du droit coutumier des Komis demeure profane et règlemente les relations interpersonnelles sur les questions liées à la forêt. Nous allons décrire ci-dessous, sur la base des thèmes qui ont émergé lors de nos terrains, la nature du droit des chasseurs komis, en y intégrant également les informations que nous apportent des auteurs plus anciens. Cet aperçu n'est pas exhaustif, mais il permet de comprendre le fonctionnement régulatoire de la chasse komie.

Comme nous l'avons noté ci-dessus, une expression imprécise et un style de narration vague sont habituels chez les chasseurs komis. En même temps, il n'y a pas réellement de raison de mentir sur toute une série de questions, comme les saisons de la chasse ou le respect des bornes des territoires. Par exemple l'un de nos amis komis a ainsi réfléchi sur les dates de la chasse aux oiseaux aquatiques, reconnaissant la réglementation existante, mais la critiquant sur la base de la logique du droit coutumier :

Aujourd'hui, en allant à Syktyvkar, j'ai vu sur la route deux sarcelles. La saison officielle de la chasse aux oiseaux aquatiques commence le 5 mai. Mais cette réglementation est mal faite. Il faudrait déterminer uniquement la fin de la saison, quand les oiseaux commencent à couver. Le début de la chasse pourrait être libre : on devrait pouvoir chasser les oiseaux dès leur arrivée. Sinon, quand la saison officielle commence, les oiseaux sont déjà partis vers le Nord. Mais dans les villages de la Parma, il n'y a pas de saison officielle... (Vassili, 40 ans ; TT, 2007) ${ }^{2}$.

La manière ici proposée de réglementer la chasse estivale est logique, et elle peut être mise en œuvre dans le contexte de certaines régions, mais elle ne convient pas pour une réglementation au niveau de l'État, car les inspecteurs de la chasse n'auraient pas les moyens de fixer partout le début précis de la couvaison. Ici transparait l'idée que les chasseurs savent eux-mêmes mieux que personne quand est la meilleure période pour la chasse et si on part du principe que les véritables chasseurs ont une éthique, la saison se réglementerait toute seule (dans ce cas, dans les villages de la Parma). Jadis, les saisons de la chasse étaient établies par les chasseurs eux-mêmes conformément aux circonstances (Bunakov, 2010 [1857], p. 34 ; Arsenjev, 2010 [1873], p. 49 ; Popov, 1874, p.73; Volkov, 2010 [1879], p. 56), mais de nos jours la suppression de toute réglementation étatique susciterait sans doute un chaos tout à fait légitime. Le deuxième message dans cette histoire est que la réglementation officielle est trop unique et que, suivant les années, les circonstances peuvent changer de sorte que le début de la saison est placé de manière si peu pertinente que la période réellement fonctionnelle pour la chasse se termine avant que la saison officielle commence.

Les règles de droit coutumier des chasseurs komis sont claires et logiques et reposent dans les cas les plus généraux sur les spécificités des saisons et sur le mécanisme 
héréditaire de partage des terres, comme elles ont été documentées dans les sources les plus anciennes. Konakov, par exemple, décrit (1983, p. 166-169) la propriété commune de la forêt conformément au droit coutumier et la répartition de celle-ci en zones de chasse individuelles pour la chasse d'automne, zones destinées au travail individuel et qui se transmettent en cas de décès du chasseur, à son fils cadet. Mais pour la chasse collective d'hiver la région appartenait au premier groupe arrivé sur place, qui avait construit une cabane ou un abri et qui avait commencé à y chasser (Konakov, 1983, p.171). Le même principe de propriété pour la forêt est toujours valable parmi les chasseurs komis aujourd'hui :

Pour les Komis du Nord, la forêt est commune. Certes, elle se divise en zones de chasse, mais de manière générale, à d'autres périodes, elle est commune. En septembre la forêt est encore divisée en zones de chasse, ce qui veut dire que chacun doit chasser dans sa zone, mais après Pokrov ${ }^{3}$ on peut chasser dans toute la forêt. On chasse là où le chien vous conduit, sur les traces du gibier. Bien sûr, [on chasse quand même] à des endroits précis, mais quand votre chien vous conduit ailleurs, alors on chasse ailleurs. La forêt est considérée comme propriété commune. On essaye quand même de chasser dans sa zone. Mais il arrive que votre chien vous emmène ailleurs, en suivant les traces (Oleg, 31 ans ; TT, 1996).

C'est là un principe relevant du droit coutumier, mais qui entre en contradiction avec la législation officielle. Conformément à cette dernière, la forêt appartient à l'État, mais pour les chasseurs elle est avant tout à eux. Au début de l'automne, on pratique surtout la chasse aux oiseaux à l'aide de pièges (principalement pour attraper la gélinotte), ce qui justifie que l'on reste dans une zone précise. Or après Pokrov on chasse surtout avec un chien et un fusil, un système mobile qui aussi justifie la suppression dans la pratique des bornes entre les zones de chasse.

Les chasseurs komis s'efforcent de marquer aussi peu que possible les sentiers et les zones de chasse. Bien que ce soit une manière claire de marquer les droits (Konakov, 1983, p. 167), la principale garantie des droits de chasse est la tradition vivante dans la communauté des chasseurs. Ils savent à quelle famille ou à quel chasseur individuel appartient telle ou telle zone et, idéalement, cela devrait suffire. Le marquage des sentiers a aussi pour but d'éviter de s'égarer. Mais un chasseur expérimenté connait ses sentiers même sans marquage. Il n'en reste pas moins que le marquage est pertinent, surtout pour les nouveaux sentiers. et comme personne n'est jamais sûr de ne jamais s'égarer, les marques sur les arbres servent en principe de repères. Le marquage des sentiers est aussi utile quand quelqu'un loue son sentier à un autre chasseur. Dans ce cas, celui qui loue le sentier connaît grâce aux marques l'itinéraire du sentier qu'il a loué. Les Komis eux aussi, encore au xix siècle, ont loué aux Mansis des aires de chasse de l'autre côté de l'Oural pour la chasse hivernale. Latkin (1853, p. 80) précise que quand les Komis se rendaient en Sibérie occidentale pour des expéditions de chasse, ils payaient une location de terres aux Mansi en munitions ou en outils domestiques. Or, quand on loue un terrain de chasse à des centaines de kilomètres de distance il n'est pas réaliste que l'on connaisse parfaitement l'environnement, surtout en tenant compte du fait que l'on y séjourne seulement deux ou trois mois par an. Dans ces conditions, les Komis avaient clairement intérêt à ce que les Mansis marquent leurs zones de chasse correctement. En même temps, le marquage des sentiers va de pair avec le problème des vols, qu'il favorise :

On n'essaye pas particulièrement de marquer. Surtout là où passent beaucoup de gens. Dans une petite aire de chasse, je ne marque rien du tout. Je connais le chemin. et c'est là qu'il y a eu moins de vols. J'y passais un jour sur deux. 
Aujourd'hui j'y vais, demain je me repose et après-demain j'y retourne. Beaucoup de gens passent par là. On peut faire confiance, mais il vaut mieux contrôler. On essaye de marquer les endroits où on passe peu, des gens qui vont ramasser des baies ou d'autres (Oleg, 31 ans ; TT, 1996)

Pour différentes raisons-amélioration des conditions de transport, importance économique grandissante de la collecte de baies et de champignons, transformation des communautés-de plus en plus souvent des gens sans rapport avec la chasse traditionnelle se retrouvent dans les aires traditionnellement utilisées par les chasseurs. L'ignorance des règles du droit coutumier ainsi que parfois la malveillance ou la négligence expliquent que parfois les pièges d'autrui sont dévalisés. Ainsi, quand les chasseurs décident quand même de marquer leur sentier, ils essayent de le faire de manière à ce que le marquage ne saute pas aussitôt aux yeux d'un passant de fortune. Le marquage ou la maîtrise des sentiers est important aussi parce qu'à proximité des villages les sentiers de différents chasseurs peuvent se croiser. Dans ce cas il faut respecter les droits et les intérêts les uns des autres :

Je suis passé au moins six fois par des sentiers d'autres chasseurs. Ces sentiers n'étaient pas parallèles, ils se croisaient. Les autres voient bien qu'un petit garçon va chasser. Je n'ai touché à rien. Ce sentier, c'est C qui me l'a donné, il appartenait à son grand-père. Son grand-père était déjà âgé, il restait près du village. Ce sentier passait trois fois par d'autres sentiers. Je n'ai jamais posé des pièges à proximité des autres sentiers, sur une distance de cent mètres, pour que les autres ne se fâchent pas. Une fois je n'ai pas fait attention. Il y avait là toutes sortes de sentiers - des sentiers pour les chevaux et pour les vaches entre autres. Avant, dans la forêt, autour du village, il y avait un enclos où on mettait les vaches. Je me suis dit que c'était un sentier pour les chevaux. et j'ai mis mon piège juste au croisement ! Ce voisin, maintenant, il est mort, il était tractoriste. Il m'a brisé mes pièges. J'ai pensé alors que les chevaux les avaient piétinés. Plus tard j'ai compris et je n'y ai plus jamais mis de pièges. J'en ai mis ailleurs, sur le sentier à chevaux. J'y reviens, mon piège a été jeté par terre. La même chose s'est reproduite une deuxième et une troisième fois. Après j'ai vérifié, un crin de cheval était resté à proximité. C'était clair, c'était un sentier emprunté par les chevaux. Ils vont dans la forêt pour échapper aux insectes. Ils se frayent des sentiers spéciaux tels que les branches puissent les débarrasser des simulies. Les vaches font la même chose, elles se fraient des sentiers dans fourrés, là où il y a des arbres et des hautes herbes (Vassili, 35 ans, 2002)

Ces réflexions impliquent que le mode de communication préféré des chasseurs komis est de suggérer par des actes à une personne qu'elle a commis une infraction (dans ce cas, en brisant un piège mal placé) (voir Konakov, 1983, p. 170). Cette communication indirecte peut cependant susciter des confusions, notamment dans le cas où les gros animaux domestiques ont utilisé aussi le droit de se frayer des sentiers. La morale de cette histoire est qu'il faut tenir compte sur les sentiers de chasse des autres partenaires possibles (chasseurs et animaux), qui ont dans la même zone des droits de circulation.

Quand, à l'automne, on établit le droit de chasser et la propriété du gibier d'après les sentiers, pour ce qui est de la saison d'hiver, les membres des artels de chasse laissent des traces de skis dans la forêt autour de la zone où ils entendent chasser. Les membres des autres artels n'ont pas le droit de franchir cette ligne. Le gibier abattu à l'intérieur de cette aire appartiennent aux membres de l'artel qui ont laissé la trace (Konakov, 1983, p. 171 ; Il'ina, Ul'ašev, 2009, p. 117). La question de savoir à qui revient le gibier est 
régie dans le droit coutumier komi par tout un ensemble de règles. Nos amis aussi aiment discuter sur la mise en place des normes de propriété du gibier :

Avant il y avait encore une grande quantité de toutes sortes de règles. Disons, un élan ou un ours a été abattu. Si quelqu'un venait au moment où on n'avait pas commencé à dépecer l'animal, il avait lui aussi droit à une partie. Maintenant je ne sais pas. Sans doute pas. Si on abattait un animal qui appartenait à quelqu'un d'autre, il fallait le redonner à son propriétaire. Si on tombait sur un animal blessé, il appartenait au premier qui avait infligé une blessure (Oleg, 31 ans ; TT, 1996)

Le narrateur renvoie ici en même temps aux règles de chasse transmises oralement et à celles fixées par écrit dans la littérature (voir par exemple Konakov, 1983, p. 172-174) qu'il connaît. Les chasseurs komis aiment à lire des revues et des œuvres de référence spécialisée, ainsi que des textes scientifiques, c'est pourquoi il n'est pas toujours facile de savoir d'où proviennent certaines informations concrètes. En même temps la règle d'après laquelle celui qui survient avant le dépeçage reçoit une partie de la viande est très spécifique et aujourd'hui les conditions pour son application ne sont sans doute pas réunies. Mais de manière générale les règles de propriété et de partage du gibier notées par les chercheurs dans le passé (que l'animal appartient à celui à qui revient la zone dans laquelle il a été chassé ou encore qui lui a infligé la première blessure, ou encore qui a commencé la poursuite) sont toujours en vigueur de nos jours.

Derrière la question de la propriété du gibier (de même que toutes les règles de droit coutumier) on retrouve la question éternelle de l'honnêteté, à laquelle l'un de nos amis renvoie également :

Avant, les gens étaient bien, honnêtes. Il m'est plusieurs fois arrivé que quelqu'un trouve dans la forêt un oiseau dans mes pièges. Il le prend, le couvre pour que la pluie ne l'endommage pas, puis remet en place le piège. Le temps que j'aille relever mes pièges, il se peut qu'il y en ait deux. Les femmes vont ramasser des baies, et quand elles voient des oiseaux dans les pièges, elles agissent de même. Elles me connaissaient, j'habitais à côté (Vassili, 35 ans, 2002).

Vassili renvoie à la règle komie de la forêt, qui prescrit l'appartenance du gibier au propriétaire du piège, du sentier ou de l'aire de chasse ou à son utilisateur autorisé (voir Balov, 2010 [1840], p. 15 ; Bunakov, 2010 [1857], p. 35 ; Arsenjev, 2010 [1873], p. 43 ; Popov, 1874, p. 49 ; Volkov, 2010 [1879], p. 57 ; Nalimov, 2010 [1924], p. 79-80 ; Il'ina, Ul'ašev, 2009, p. 117). En principe, un chasseur qui trouve du gibier dans le piège d'un autre chasseur peut le prendre et l'apporter à son légitime propriétaire, mais il est préférable que le propriétaire le rapporte en personne. En même temps, quand un piège se trouve à un endroit facilement accessible à des inconnus, il peut être pertinent de rapporter le gibier le plus rapidement possible (TT, 2003).

Chez les chasseurs komis il est depuis des temps ancestraux coutume qu'en l'absence du propriétaire d'une cabane de chasse, les visiteurs (d'autres chasseurs ou d'autres voyageurs, par exemple, des prisonniers évadés) peuvent passer la nuit et utiliser tout ce qui s'y trouve, par exemple la nourriture. En remerciement et en compensation, il convient de laisser en partant quelque chose d'utile en cadeau pour le maître de maison et s'il est possible ou nécessaire une indication sur la personne qui y a séjourné. Il convient de laisser la cabane en bon ordre et de vérifier si possible que le propriétaire ait les réserves nécessaires pour vivre (Popov, 1874, p. 49 ; Konakov, 1983, p. 176-177). C'est là une règle générale de la forêt, qui est naturelle encore aujourd'hui pour les chasseurs komis. 
32 À maintes reprises les chasseurs komis nous ont raconté des cas où on a fait appel aux normes du droit coutumier pour résoudre des conflits entre chasseurs et travailleurs de l'industrie forestière. Un hiver, deux amis chasseurs, Igor et Jegor, étaient déjà dans la forêt depuis quelques mois. Igor est tombé gravement malade. Il avait de la fièvre, il n'arrivait pas à chasser et Jegor devait s'occuper de lui, de sorte que lui non plus n'a pas $\mathrm{pu}$ chasser suffisamment. Au bout de quelques temps, leurs réserves de nourriture arrivèrent à bout. Comme à proximité travaillaient des ouvriers de l'industrie forestière, ils ont décidé d'aller leur demander de l'aide. Quand Igor et Jegor arrivèrent à leur wagon, celui-ci était verrouillé et les ouvriers étaient rentrés chez eux pour le week-end. Étonnés de ce que le wagon était verrouillé, ils défoncèrent la porte et pénétrèrent à l'intérieur. Mais ils n'étaient pas au bout de leurs surprises : en effet à l'intérieur chaque ouvrier avait son armoire et toutes étaient verrouillées. Cela dépassait leur entendement : pourquoi fermer ses affaires vis-à-vis de personnes avec lesquelles on partage le même local de vie. Les chasseurs ouvrirent tous les verrous et vécurent le week-end sur les vivres des ouvriers en l'attente du car qui ramènerait les ouvriers dans la forêt et qui pourrait les ramener au village. Quand les ouvriers furent arrivés, indignés, ils protestèrent. Alors Igor tira un coup de fusil devant leurs pieds et leur fit la morale expliquant comment on vit dans la forêt, qui est le maitre des lieux, sans oublier de dire haut et fort ce qu'il pense de la manie des ouvriers de tout verrouiller, qui montre qu'ils n'ont aucune confiance l'un dans l'autre.

Tout laisser à porter dans la main dans une cabane dans la forêt est essentiel pour que les personnes en difficulté puissent trouver ce dont ils peuvent avoir besoin. Le respect mutuel et l'assistance mutuelle sont indispensables dans la vie des chasseurs pour garantir la survie et pour éviter les problèmes. Le partage des ressources entre chasseurs est l'un des éléments de leur mécanisme d'adaptation. L'attention portée aux visiteurs de fortune est effectivement une loi très ancienne, comme le confirme par exemple Latkin (1853, p. 78) qui raconte que les pêcheurs komis avaient pour coutume de toujours offrir du poisson à tout passant le long de la rivière.

Mais dans le dernier cas relaté on peut aussi lire une allusion à la dégradation de la morale de la chasse ou, plus généralement, de l'éthique de la communauté. Ce thème est l'un des plus anciens et des plus stables aussi bien dans l'histoire des observations ethnographiques que dans l'oralité (Lipin, Leete, 2015, p. 25-28). En rapport avec la chasse komie, il est question de dégradation générale ainsi que de disparition de l'honnêteté dès les premières descriptions détaillées (voir par exemple Latkin, 1853, p. 48-50 ; Nalimov, 2010 [1903], p. 26 ; Abramov, 1914, p. 57). Vassili Latkin (1853, p. 50) raconte dans son journal de terrain des années 1840 son séjour chez les Komis, où il dit qu'avant, personne ne volait dans la forêt, mais que la famine avait eu des effets délétères sur l'éthique collective. Arsenjev lui aussi se plaint de ce qu'avant les Komis ne connaissaient pas le vol ou le mensonge, mais que la famine et l'oppression administrative avaient conduit à la multiplication de ces pratiques «dans une assez large mesure » (Arsenjev, 2010 [1859], p. 40 ; 2010 [1873], p. 42-43).

Pourtant les auteurs du XIXe siècle sont largement d'avis que l'honnêteté chez les Komis et surtout chez les chasseurs komis est toujours dominante et que c'est une valeur, au moins jusqu'à une période très récente, indiscutée, comme le remarque Arsenjev (2010 [1873], p. 43). D'autres auteurs de l'époque tsariste (Popov, 1874, p. 49 ; Volkov, 2010 [1879], p.63-64; Abramov, 1914, p. 35) renvoient aussi à la fameuse «honnêteté zyriène ». Abramov présente un vieil homme komi, Vassilij Ivanovič, appelée en komi 
Čikči Vass. D'après ce dernier la cause de la dégradation de la vie komie est que les Komis ont tourné le dos à la foi et à la vérité. Ce rejet de la vérité couvre aussi le détournement de l'éthique de la chasse - en raison de leur cupidité, les chasseurs se sont mis à attraper plus d'animaux et d'oiseaux qu'ils n'en avaient besoin, ou encore par rapport à l'équilibre de l'environnement, ce qui explique que le nombre d'animaux et d'oiseaux dans les forêts komies a considérablement diminué (Abramov, 1914, p. 53). La question de l'honnêteté des Komis relève aussi du processus d'ensemble de transformation culturelle - réel et imaginaire. Le recul de la connaissance et du respect des normes coutumières archaïques se produit aussi dans la réalité, mais le changement permanent du système social de référence amène à poser la question de la dégradation et de l'honnêteté de manière ambivalente. Pour les chasseurs komis, le recul de la reconnaissance de l'honnêteté et du droit coutumier sont une réalité culturelle centrale. En même temps, la dégradation culturelle est indiscutablement un des motifs les plus répandus d'interprétation de l'activité humaine. Les gens sont habitués à percevoir l'évolution des choses par l'intermédiaire de ce prisme critique.

Les transformations sociales ont apporté aux chasseurs dans la forêt par exemple les ouvriers forestiers, qui vivent provisoirement dans la forêt pour abattre les arbres et les transporter ailleurs. Les conceptions des ouvriers à propos des relations de propriété et de l'étiquette des comportements interpersonnels demeurent quelque peu difficiles à comprendre pour les chasseurs. Ceux-ci sont en même temps convaincus que dans leur loi de la forêt est valable pour tous et que personne n'a le droit de l'enfreindre, y compris ceux qui sont là de manière provisoire, qui pensent vivre à part. Or personne dans la forêt n'est jamais pleinement isolé des autres, tout un chacun peut se retrouver dans le besoin et les possibilités d'assistance peuvent être limitées.

En même temps on peut trouver d'autres avis sur la question de l'honnêteté komie. Par exemple Evgenij Balov (2010 [1840], p. 15) considère qu'une certaine forme de tromperie est, chez les chasseurs komis, habituelle. D'après ses matériaux, des bardeaux à l'intérieur des peaux d'écureuil sont oints de sang chaud, puis on refroidit les peaux et on les malaxe de sorte que l'intérieur en devienne blanc. Les peaux ainsi travaillées étaient vendues plus cher en raison de leur belle couleur, car la couleur blanche de l'intérieur est censée être un signe de la qualité de la peau. Or les peaux sont vendues retournées, de sorte que la valeur de la peau est déterminée par son aspect intérieur (idem, p. 16).

39 Cet exemple pourrait suggérer que l'honnêteté pour les chasseurs komis ne concerne pas le processus du marchandage. En même temps Konakov (1983, p. 178) affirme que les chasseurs komis étaient réputés parmi les marchands pour leur honnêteté. Il faut donc reconnaître que la question de l'absolue honnêteté des chasseurs komis dans la vente de leurs produits reste contradictoire. Mais même si les chasseurs s'accordaient certaines libertés dans la communication avec les marchands de fourrures, c'est là une question qui ne concerne pas directement la vie dans la forêt.

L'honnêteté demeure pour les chasseurs komis un principe de fonctionnement central du droit coutumier, mais on rencontre dans ce domaine des problèmes au moins depuis que la culture komie est décrite. Dès le XIX ${ }^{\mathrm{e}}$ siècle, différents auteurs ont relaté des pratiques contredisant "l'honnêteté zyriène". Et encore aujourd'hui, les chasseurs sont confrontés à différents problèmes liés aux infractions au droit coutumier. Or pour pouvoir sanctionner quelqu'un ou le remettre sur le droit chemin, encore faut-il commencer par identifier ou trouver le coupable. 


\section{Mettre la main sur le coupable}

41 même temps, la pratique même du droit coutumier en caractérise le fonctionnement dans les groupes et entre les groupes de manière bien plus éloquente qu'une énumération de principes. Les récits de chasse permettent de faire ressortir quelques moyens magiques par lesquels le responsable-sorcier de l'artel de chasse tire au clair le sort jeté par un autre artel et qui les prive de chance à la chasse (Sidorov 1997 [1928], p. 41-44). D'après cet auteur le fondement des infractions au droit coutumier venait du sentiment d'impunité des artels de chasseurs. Ceux-ci considéraient que leur responsable était sorcier et que sous sa protection il était possible d'ignorer les intérêts des autres artels ou de chasseurs individuels. D'après les conceptions traditionnelles, les responsables d'artels pouvaient également s'engager dans une bataille magique destinée à nuire à l'ensemble des expéditions de chasse de l'autre. Dans ces cas-là, on essayait d'endommager par la magie les fusils, les chiens de l'autre artel, de remplacer les véritables animaux par de faux-semblants, de faire perdre la capacité d'orientation, ou tout simplement de jeter sur l'autre artel le mauvais œil. En même temps la peur des sorciers dissuadait aussi les chasseurs d'enfreindre la coutume (1997 [1928], p. 42-49, 61). existante (Lipin, Leete, 2000). Les chasseurs komis comptent en général sur l'étroit réseau de relations, qui ne permet pas à l'auteur d'une infraction de rester anonyme. Quand on en découvre une, le cercle des suspects n'est jamais très large et il est relativement aisé d'identifier le coupable.

celui-ci n'estime pas avoir commis d'infraction, il faut commencer par l'en convaincre et pour ce faire les Komis disposent d'un arsenal traditionnel ad hoc, par exemple ils détruisent le piège de quelqu'un qui est allé chasser sur le terrain d'autrui :

Quand j'étais à l'armée, quelqu'un a ouvert à proximité de mon sentier un nouveau sentier. Je lui ai laissé une lettre de mise en garde, à laquelle il n'a pas réagi et il a continué à chasser. Alors j'ai brisé ses pièges. Alors il a brisé mes pièges. Je ne sais pas qui c'était. Et ainsi plusieurs fois. On détruisait nos pièges mutuellement. Il a fini par laisser tomber. Il a cessé de briser mes pièges et de chasser. Il a peut-être appris que j'étais dans mon droit. Sans doute quelqu'un lui a dit que c'était ma zone à moi. J'allais souvent ramasser des baies et j'avais vu que personne ne chassait sur ce vieux sentier depuis dix ans. C'est ainsi que j'ai obtenu ce sentier, et que j'y ai mis mes propres marques (Oleg, 32 ans; TT, 1997)

Dans ce cas précis, le contentieux sur le sentier s'est déroulé de telle manière que les parties ne se sont jamais rencontrées et que le chasseur à qui le sentier appartenait n'a jamais su qui, entre-temps, avait occupé son sentier. Finalement le conflit s'est résolu de manière pacifique et le chasseur à qui, d'après le droit coutumier, le sentier appartenait, est rentré dans ses droits. D'ailleurs les deux chasseurs avaient utilisé le sentier de manière analogue, utilisant un endroit qui, à leur avis, était abandonné. D'après la coutume komie, un chasseur ne perd pas le droit à son sentier même s'il ne l'utilise pas pendant longtemps (Konakov, 1983, p.170), ainsi, le narrateur avait davantage droit au sentier que son concurrent. L'occupation du terrain est, avec l'héritage ou la location, une troisième manière de devenir, en droit coutumier, propriétaire d'un sentier de chasse.

Études finno-ougriennes, 49-50 | 2018 
même chasseur a raconté un autre cas d'infraction au droit coutumier de la chasse, laquelle dans ce cas a été commise à l'aide de la magie. Le narrateur affirme qu'il était enfant, en 1979, quand il a été témoin de cet acte magique :

Un jour, nous étions ensemble, avec un voisin. Un autre voisin volait : il allait sur des sentiers d'autrui et notre voisin s'en est rendu compte. J'ai deux voisins. L'un manifestement était allé aux champignons et il a remarqué que l'autre volait dans la forêt. Mais le propriétaire du sentier, lui, n'en sait rien. Le voisin agissait de sorte que quand on s'approchait, on croyait que des bêtes étaient passées par là, pas des hommes. J'avais quatorze ans et j'ai entendu leur dialogue. Et ce voisin demande :

- Comment ça va? Ton sentier te rapporte quelque chose?

L'autre dit:

- Pas grand-chose. Quand j'arrive, les bêtes et les oiseaux ont tout mangé. Il est rare que quelque chose tombe dans les pièges. Ou plus précisément cela arrive, mais ils ne restent pas.

L'autre dit :

- Apporte-moi une bassine d'eau. Là, vous avez une bassine en cuivre pour vous laver le visage. Apporte-la-moi. Avec de l'eau. Je vais regarder et te dire qui est le coupable.

L'autre dit alors à son voisin :

- Tu as perdu la tête? Tu vas regarder dans la bassine? Il y a de l'eau, rien d'autre.

Il a pris cette demande pour une plaisanterie, mais il lui a quand même apporté la bassine. Le voisin a regardé :

- Là, je vois qui est le coupable. Je vais lui dire et il te rapportera aussitôt ce qu'il t'a pris. S'il ne l'a pas encore mangé, il te le rapporte.

Et il est parti. Environ une demi-heure plus tard il rapporte de l'autre rue un coq de bruyère et un tétras et dit :

- J'étais aux champignons, j'ai vu qu'ils n'allaient pas tarder à pourrir. Je les ai pris.

Il n'a pas dit qu'il les avait volés. Il a dit qu'il ramassait des baies. Mais pourquoi alors il a attendu si longtemps pour les rapporter? Mais il est a quand même rapportés. Après, j'ai demandé au voisin qui avait regardé dans la bassine :

- Comment tu t'y es pris?

Il m'a dit qu'il avait vu comment l'autre avait pris les oiseaux. Alors il était allé le voir et lui avait dit :

- Avec Dima, j'ai regardé dans l'eau d'une bassine en cuivre et le regard est tombé sur toi. C'est toi qui étais dans la bassine.

L'autre a pris peur et dit :

- Pas possible! Comment ça?

- Nous avons regardé comme dans un miroir. Tu y étais, tu prenais deux tétras.

Donc, il l'a effrayé. Et l'autre est venu et les a rapportés entiers. C'était il y a longtemps. Maintenant, ce n'est plus possible de faire peur à quelqu'un de la sorte (Oleg, 33 ans ; TT, 1998).

Même si ici le narrateur a convaincu le voleur de reconnaître son acte par tromperie, l'acte de regarder l'eau dans la bassine en cuivre a été perçu par le voleur comme une utilisation de la magie. Ainsi, cette manière d'identifier l'infraction a été en même temps aussi un châtiment (car plusieurs personnes ont été ainsi informées du vol, cette histoire s'est répandue) et un acte prophylactique - s'il est tellement facile d'identifier un coupable à l'aide de la magie, les gens sont moins enclins à voler.

Il arrive cependant qu'il ne soit pas aussi facile de mettre la main sur le coupable; celui-ci peut aussi en dépit de pièces à conviction ne laissant aucun doute, mettre en cause le droit du propriétaire du sentier :

Une fois, j'ai attrapé un homme. J'en avais assez, les vols étaient permanents. Un matin j'ai pris un fusil, je suis allé me tapir dans les fourrés à proximité d'un piège. Et je vois, un homme arrive avec son fils. Mais moi, j'ai une gélinotte dans le 
piège. Il l'a vue, elle était bien visible. Et moi j'étais aux aguets dans les buissons. Il s'approche et sans même regarder autour de lui, hop ! il s'en empare.

Moi, je sors mon fusil et je lui dis :

- Remets-la tout de suite où tu l'as prise !

Et lui se met à discuter avec moi :

- Qui tu es, toi?

- Remets-la tout de suite en place! Ce n'est pas toi qui as fait ce piège. C'est moi qui l'ai posé. Remets tout de suite l'oiseau à sa place !

Il n'obtempère pas! Alors je tire un coup de fusil à un mètre de lui. Alors il l'a remise en place, il a arrêté de discuter. Je lui dis :

- C'est tout. Que je ne te revoie plus.

Il venait du bourg. Je ne sais pas s'il était komi ou russe. Quelle différence ? Je lui dis :

- Tu es là avec ton fils. Il a neuf ou dix ans. Quel exemple tu lui donnes? Il va se mettre à voler comme toi ?

C'est ce que je lui ai dit et il s'est mis à me crier dessus :

- Pourquoi, elle est à toi la forêt?

Moi je lui dis :

- C'est moi qui ai fait ce piège. Pas toi. Cela fait des années que je viens ici. et toi tu viens et tu te sers. Pourquoi tu te comportes ainsi?

Et lui me crie dessus... Il n'a même pas peur. Je lui dis de remettre la gélinotte en place et il n'a rien fait tant que je n'ai pas tiré. Il m'insulte comme si je n'avais pas un fusil à la main. Mais dès que j'ai tiré tout est rentré dans l'ordre. Il a vite couru, il a remis la gélinotte en place (il rit). Comme ça, c'est amusant, mais quand on est là... Maintenant c'est amusant, mais sur place c'était douloureux. (Oleg, 33 ans ; TT, 1998)

Le sentier du narrateur se trouvait à proximité du village, juste à quelques kilomètres des maisons. Lui-même allait relever les pièges un jour sur deux, pour que les villageois n'aient pas le temps de beaucoup voler. À proximité il y avait aussi de bons emplacements à champignons et à baies, ainsi, à l'automne, quand les saisons de la gélinotte, des baies et des champignons coïncidaient, il y avait beaucoup de circulation. et beaucoup de ceux qui circulaient dans la forêt n'avaient pas la moindre idée, ou ne voulaient pas avoir la moindre idée du droit coutumier. De ce fait, les chasseurs étaient obligés pour se protéger d'utiliser des méthodes musclées telle que celle que le narrateur vient de relater (voir aussi Iljina, Uljašev, 2009, p. 117-118). En fait, il ne faut même pas vraiment avoir des connaissances particulières en droit coutumier: si quelque chose de trouve dans un piège, le piège a vraisemblablement un propriétaire et s'est donné du mal pour le mettre en place. Ainsi, les chasseurs ne voient aucune raison d'excuser les voleurs.

Comme le souligne le narrateur de ces derniers cas, les infractions, la découverte du coupable et la solution trouvée peuvent être en même temps amusantes et lamentables. Il peut aussi arriver que le chasseur n'ait aucun mal à trouver le coupable, mais que l'absurde de la situation ne permette pas de la résoudre:

Igor est allé à la chasse, en voiture, tirer les lièvres la nuit. El est une marque de voiture étrangère, c'est pourquoi Igor voulait aller à la chasse en UAZik, pour pouvoir tirer depuis la fenêtre en plein mouvement. Ils étaient partis cinq heures. Ils reviennent et racontent qu'ils ont vu six lièvres. Trois d'entre eux étaient trop loin, de sorte qu'ils ont eu le temps de s'enfuir dans les buissons. Dans trois cas, leur voiture a été doublée et les autres ont tiré le lièvre sous leur nez. Les policiers ont pour habitude de doubler au dernier moment, de tirer le lièvre et de le récupérer. Eux aussi, ils chassent. Igor avait été sorti du lit au milieu de la nuit pour aller à cette chasse. Ils sont revenus, ils étaient tout mouillées et frigorifiés ... (Larissa, 37 ans ; TT, 2007). 

celui-ci a trouvé le gibier, ils l'ont tiré eux-mêmes. Logiquement, il aurait fallu dans ce cas infliger une sanction à ceux qui ont violé le droit coutumier (d'après le principe que le gibier appartient à celui qui le trouve, même avant que celui-ci le tire), mais quand les voleurs sont les gardiens de l'ordre, cela n'a pas grand sens de chercher à tirer vengeance. La police a bien suffisamment d'occasions de chercher noise aux chasseurs utilisant les possibilités donnés par leur position ou par la législation. Mais en principe, si on laisse de côté ce point de rencontre délicat entre le droit officiel et la pratique coutumière, la tradition komie prévoit différentes manières de châtier les coupables.

\section{Les châtiments}

C'est principalement à l'intérieur de la communauté que la force normative de la coutume s'exerce, mais cela suscite aussi des attentes juridiques et morales allant dans le sens de la reconnaissance de ces règles dans un contexte social plus large (WIPO, 2013, p. 12). En même temps, l'autorité punitive du droit coutumier peut ne pas suivre ces attentes de manière aussi efficace en dehors des frontières de la communauté. Leur application est d'autant plus sérieuse dans le milieu des chasseurs.

Comme nous l'avons dit, le châtiment le plus simple et en même temps non moins efficace pour une infraction au droit coutumier, passe par la parole : le coupable reçoit un sobriquet lié à son infraction et la connaissance de celle-ci se répand dans la communauté. Aujourd'hui, les gens ne craignent plus forcément autant cette réputation, car les villages s'agrandissent et deviennent de plus en plus hétérogènes en raison de l'arrivée des nouveaux-venus. Ainsi, ce mécanisme traditionnel de contrôle social ne fonctionne plus de manière aussi efficace qu'avant. En plus de l'attribution d'un sobriquet et de la diffusion de l'information, qui sont le châtiment de base, pour chaque cas de figure viennent s'ajouter des peines diverses, par exemple si quelqu'un a construit une cabane sur une terre libre, mais trop proche de la cabane d'un autre chasseur, on peut lui brûler sa cabane. D'après la tradition des chasseurs komis, personne ne lui dira rien. Et tant qu'il sera dans sa cabane, il ne lui arrivera rien. Mais dès qu'il la quitte, elle sera brûlée. Et bien sûr, l'histoire sera racontée et le chasseur se verra affublé d'un sobriquet (voir par exemple Konakov, 1983, p. 170-173). Une fois de plus nous voyons que tant que c'est possible, les chasseurs komis ne parlent pas directement de l'infraction avec son auteur mais ils y font allusion (dans les cas mentionnés ci-dessus, il s'agissait de pièges brisés, ici de cabane mal placée brûlée).

L'approche qui consiste à laisser construire la cabane, y laisser le chasseur y vivre pendant une saison et n'appliquer la peine qu'après semble renvoyer à une brutalité traditionnelle qui va de pair avec le droit coutumier komi. Il peut en effet arriver que l'infraction soit commise de bonne foi en toute ignorance, et son auteur pourrait avec une allusion en temps et en heure, s'en rendre compte. Mais cette brutalité est inscrite dans les pratiques coutumières komies. Les Komis partent du principe qu'un chasseur qui opère dans la forêt connaît suffisamment les règles, ou, s'il ne s'agit pas de quelqu'un originaire de la région, qu'il étudie la situation avant d'agir. Si quelqu'un fait quelque chose de travers, il en est entièrement responsable personnellement, et aucune circonstance atténuante ne lui est réservée. Les chasseurs ont eux aussi insisté sur cet aspect de dureté dans leurs conversations avec nous :

Études finno-ougriennes, 49-50 | 2018 
Avant, les châtiments qui accompagnaient le vol étaient très sévères. On pouvait même tuer si... Mais en général, à ce qu'on raconte, je ne sais pas, on l'attachait à un bâton. On faisait passer un bâton par les manches et on laissait partir la personne. Quand on attrapait le voleur, c'est ainsi qu'on le punissait. En général quand on apprenait que quelqu'un volait, on le suivait et on l'attrapait. Au village en général les gens se connaissaient. La confiance était détruite. (Oleg, 31 ans ; TT, 1996) suspendre par les cheveux le coupable nu à des branches d'arbres entrelacées (Sidorov,
1997 [1928], p. 267 ; Konakov, 1983, p. 173 ; Il'ina, Uljašev, 2009, p. 121). Il était privé de
son couteau et ne pouvait aucunement se libérer. D’après Sidorov (1997 [1928], p. 267) bien d'autres châtiments débouchaient sur la mort. Parfois le voleur était brûlé avec sa cabane de chasse, ou encore on lui laissait dans la cabane un gâteau empoisonné. D'après la loi de l'hospitalité, l'inconnu a droit de prendre ce qu'il trouve dans la cabane de chasse. Mais quand on sait que celle-ci est fréquentée par un voleur, par un contrebandier, on lui laisse de la nourriture empoisonnée : il suffit qu'il la mange et il meurt. Le gâteau empoisonné pouvait aussi être laissé dans la cabane de chasse du voleur en guise de remerciement pour l'utilisation de la cabane. À l'époque tsariste les juges n'essayent pas particulièrement de résoudre ce genre de cas. En tout cas, personne ne dénonçait jamais personne (voir aussi Konakov, 1983, p. 173 ; Il'ina, Uljašev, 2009 : p. 121). Si pendant une chasse quelqu'un s'enfuyait, laissant les autres dans une situation dangereuse, par exemple à la merci d'un ours blessé, le coupable était privé de sa part et était chassé de l'artel, voire du village. On pouvait aussi endommager le fusil du voleur, ou encore empoisonner son chien, ou encore l'abandonner dans la forêt sans pantalon pendant la saison des moustiques (Mihailov, 2010 [1851], p. 22 ; Žakov, 1990 [1901], p. 324 ; Konakov, 1983, p. 170-171, 177 ; Il'ina, Uljašev, 2009, p. 114, 121).

57 Un de nos interlocuteurs confirme l'existence jadis de règles très strictes :

Aujourd'hui, il n'existe plus rien de pareil. Aucune morale générale. Juste un peu.

L'un suit telle règle, l'autre non (Oleg, 31 ans ; TT, 1996).

Aujourd'hui aussi les gens sont conscients sur le principe que le fonctionnement correct de la chasse implique des châtiments sévères, mais ceux-ci sont devenus moins durs. En général le voleur est tabassé, ou bien il ne se passe rien, car les infractions sont 
devenues fréquentes et que le coupable n'a pas été identifié, comme cela arrive souvent.

La brutalité des châtiments s'applique aussi aux chiens, que les maîtres laissent circuler librement dans la forêt autour du village et qui souvent vident les pièges. Les chasseurs komis sont d'avis que quand un chien commence à enfreindre les coutumes, il est impossible de le dresser et la seule solution est de l'abattre :

Au village, on n'abat pas son chien. Mais dans la forêt il faut le faire (oleg, 41 ans ;

TT, 2006 ; voir aussi Leete, 2011, p. 170-171).

Cela veut dire que quand on rencontre dans la forêt le chien d'un chasseur négligeant, on a le droit de l'abattre. De même, comme dans le cas des personnes, la brutalité est en paroles beaucoup plus grande que dans la réalité, car bien que pour ce qui est de la nécessité d'abattre les chiens, les chasseurs sont résolus (aussi dans le cas d'un chien personnel qui ne se comporte pas en bon chien de chasse), il est quand même rare que la peine capitale soit appliquée. Par exemple d'après les chasseurs il faut abattre un vieux chien de chasse qui certes a pu bien servir son maître pendant des années, mais qui ne peut plus le faire car il a perdu l'odorat. Mais jamais les chasseurs ne vont plus loin que les propos. Les chasseurs estiment d'ailleurs que ce n'est pas nécessaire, car les chiens comprennent les propos des gens et quand en sa présence on évoque la possibilité de l'abattre (même si au lieu d'abattre on dit «transformer en moufle », mais le chien comprend la langue mystérieuse des chasseurs), un chien qui se respecte part seul dans la forêt mourir (TT, 2016 ; voir aussi Leete, 2011, p. 179).

61 Les chasseurs komis appliquent les règles du droit coutumier non seulement aux chiens, mais aussi aux bêtes sauvages et aux oiseaux qui se présentent comme des concurrents, qui aiment à prendre le gibier pris au piège. Parfois, les ours surveillent les sentiers de chasse et profitent d'un « déjeuner gratuit » en se servant par exemple des gélinottes prises au piège. C'est pourquoi les Komis posent sur les même sentiers, à côté des pièges à gélinotte aussi des pièges à ours. Même si l'ours n'est pas pris, ils pensent qu'il comprend qu'il n'est pas le bienvenu sur ce sentier. Parmi les autres voleurs de gibier, il faut mentionner les corbeaux - pour eux aussi, la juste rétribution, estime-t-on, est la mort. Les Komis ont leurs pratiques pour chasser les corbeaux. Par exemple ils imitent le battement des ailes d'une gélinotte prise au piège pour attirer les corbeaux (voir Lipin, Leete, 2015, p. 18-19).

Les chasseurs komis connaissent aussi les châtiments magiques, qui, d'après Uljašev, sont surtout répandus dans l'oralité (Il'jina, Uljašev, 2009, p. 121). Celui qui a commis une infraction contre le droit coutumier est menacé de perte de l'aptitude à tirer (Popov, 1874, p. 49), du gibier pour l'ensemble de la saison (Sidorov, 1997 [1928], p. 47-49) voir de la perte définitive de la chance à la chasse (Volkov, 2010 [1879], p. 64). Il y a cent ans, la croyance des Komis en la magie de chasse était forte et de notoriété publique. Cela avait des conséquences aussi juridiques, comme le confirme l'affirmation de Žakov, d'après lequel même les juristes de l'époque reconnaissaient le «mysticisme komi » (Žakov, 1990 [1901], p. 325). Encore aujourd'hui, les chasseurs komis sont prudents sur les questions de droit et d'ethique, car dans une certaine mesure la possibilité de s'attirer des châtiments magiques n'est jamais totalement exclue.

63 Le lien entre droit coutumier et magie tient à la position des Komis d'après lesquels tout le système de la chasse repose sur un certain équilibre animiste. On chasse sur ses terres en tenant compte de divers types d'équilibre à long terme. C'est la même conception de maintien de l'équilibre qui fonde les peines coutumières. Les êtres de la 
forêt (animaux et esprits), d'après les chasseurs, ont une agentivité semblable à celle des humains et de ce fait, les chasseurs n'ont pas le droit de se plaindre si l'équilibre naturel, mis en cause par une mort inutile, leur renvoie le contrecoup, comme un de nos interlocuteurs nous l'explique, de manière un peu grotesque, mais éloquente :

$\mathrm{H}$ part à la chasse à l'élan. Il marche dans la forêt, il tombe sur la tanière d'un ours. Il tue l'ours. Pour que celui-ci ne le mange pas. Une autre fois l'ours n'a rien voulu avoir à faire avec eux, il s'est enfui, il ne les a pas attaqués. Mais les chasseurs l'ont quand même chassé et tué. Il faut maintenir l'équilibre dans la nature : non pas que les chasseurs partent tout le temps chasser l'élan et abattent un ours. Parfois l'ours aussi devrait lui aussi pouvoir manger les chasseurs. (Aleksandr, 61 ans ; TT, 2007).

Bien que cette affirmation fasse appel à l'humour, elle reflète quand même la perception animiste d'après laquelle le châtiment de celui qui comment une infraction au droit coutumier ou à l'éthique de la chasse est administré par les esprits, les forces de la nature ou les animaux. Le châtiment n'est pas forcément une condamnation à mort, mais il peut se manifester en permanence. Dans toutes sortes d'échecs, les chasseurs peuvent lire un message renvoyant à une erreur, c'est-à-dire un châtiment pour une infraction commise.

65 C'est bien dans la magie komie que le lien entre l'animisme et le droit coutumier apparaît le plus éloquemment. Nous avons relaté plus haut comment un mot, une pensée ou une émotion (vomidz) imprudents suscitent une infraction magique. D'après différents auteurs, il existait aussi un autre type de magie: tomber sous l'influence de l'hystérie komie, la ševa, pouvait souvent être une forme de châtiment pour une infraction au droit coutumier. La ševa était particulièrement répandue là où la chasse occupait une place centrale dans les revenus de la population (Sidorov, 1997 [1928], p. 130-131 ; Konakov, 1983, p. 189-190 ; Il'ina, Uljašev, 2009, p. 108). D’après Sidorov (idem, p. 133) et Nalimov (2010 [1926], p. 213), l'hystérie allait de pair avec le fait que les chasseurs passaient une grande partie de l'année (la moitié de l'été et de l'automne, et presque tout l'hiver) loin de chez eux à la chasse. Jadis, la transmission de la ševa était, pour Sidorov "un phénomène juridique » en harmonie avec les valeurs morales de la population. Au début de la période soviétique la diffusion de la ševa était déjà vue comme plutôt fortuite, et le lien entre droit coutumier et magie était oublié.

À l'époque, la magie n'était pas seulement une question de vision du monde, mais un système de régulation des relations sociales (Sidorov, 1997 [1928], p. 266).

Les châtiments coutumiers des chasseurs komis reposent sur un fondement de brutalité, d'équilibre et de magie. Le fonctionnement ou le potentiel de leur principe directeur est censé dissuader les chasseurs de voler et de pratiquer toutes sortes d'infractions. Parfois, les châtiments documentés sont concrets (comme certains cas de règlement de comptes), parfois ces châtiments animistes sont vagues et se révèlent dans le long terme par une accumulation d'échecs d'origine à première vue inconnue. Ces mêmes châtiments peuvent provenir aussi bien des sorciers que des esprits de la forêt, et parfois les sanctions aux infractions au droit coutumier n'ont aucune source identifiée.

\section{Conclusion}

67 Le point de départ de notre article ont été les récits de chasse que nous avons collectés lors de nos terrains, et que nous avons complétés par des informations tirées de la littérature spécialisée, afin de placer les informations sur le droit coutumier komi 
d'aujourd'hui dans un contexte historique. Les thèmes principaux n'ont pas changé dans les deux ou trois derniers siècles, car les chasseurs ont toujours parlé des questions du droit coutumier et les principales questions restent encore posées. Le droit coutumier komi (et surtout les règles liées à la chasse) a été historiquement aussi encadré, dans une certaine mesure, par les lois de l'État. Aujourd'hui, le fossé entre le droit écrit et le droit coutumier des Komis est parfois plus perceptible, malgré le fait que le système juridique officiel a fait des efforts pour réduire les différences. Après le début $\mathrm{du} \mathrm{xx}^{\mathrm{e}}$ siècle, l'État n'aplus voulu établir une limite rigide entre la zone de compétence du droit officiel et celle des coutumes des comunautés locales. Cela ne veut pas dire que le système juridique étatique a pris totalement contrôle de la vie des chasseurs, mais certaines libertés ne sont plus marquées depuis longtemps.

Le cadre de fonctionnement du droit des chasseurs komis est régi par les convictions animistes. Aussi bien la vision du monde animiste des chasseurs que le droit coutumier sont orientés vers le maintien ou la formation d'un équilibre naturel et culturel. Cet impératif d'équilibre est si fort, que les moyens de garantir l'ordre juridique dans la forêt peuvent sembler brutaux. Cette brutalité de principe fait partie d'une stratégie de survie et est indispensable pour garantir le ton juste dans les relations des chasseurs entre eux et pour minimiser les possibilités de malveillance dans la vie quotidienne dans la forêt. Ce fondement animiste est souvent implicite. La plupart des règles coutumières tend à réguler les relations interpersonnelles et ne s'accompagne pas, en général, de renvois à des normes sacrales. Les règles de la chasse ont aussi des sources phénoménologiques et traditionnelles. Les chasseurs komis essayent de suivre les mêmes règles que leurs ancêtres et de maintenir la pratique de la chasse en harmonie avec les évolutions de l'état de l'environnement.

Les coutumes suivies aujourd'hui par les chasseurs komis sont anciennes, on peut suivre la documentation de ces traditions sur deux ou trois siècles. Il est logique de déduire que les principes du droit coutumier des chasseurs ont été semblables bien longtemps avant que les ethnographes n'aient commencé à les documenter. De nos jours, le rôle du droit coutumier et de l'animisme on partiellement changé mais en partie ils sont restés tels qu'ils étaient. Les chasseurs n'essayent pas d'inventer de nouveaux principes de droit coutumier, mais au besoin ils adaptent des détails de leur pratique juridique selon les circonstances.

La pratique du droit coutumier ne réglemente pas uniquement les relations intracommunautaires. Dans des circonstances où les conceptions juridiques des autochtones se heurtent à des approches étrangères, un dialogue complexe se met en place entre les applications locales et exogènes de la conscience juridique (droit étatique ou conceptions alternatives de droit coutumier) (voir WIPO, 2013, p. 6-8, 12, 18).

71 Le trait déterminant du droit coutumier est que c'est la communauté elle-même qui établit juridiquement certaines règles. Toutes les règles de comportement ne relèvent pas forcément du droit coutumier. Mais ce dernier touche aussi à l'animisme et aux êtres non humains, aux esprits et aux animaux. La composante animiste du droit coutumier ne dépasse en général pas les limites de la communauté, les autres ne reconnaissent au comportement en forêt aucune dimension religieuse. La dimension profane du droit coutumier s'étend plus facilement que la dimension animiste, c'est pourquoi les chasseurs attendent des étrangers tout simplement la reconnaissance de leurs règles de comportement. 


\section{BIBLIOGRAPHIE}

ABRAMOV K. Е. АБРАМОВ К. Е., 1914, В ЗЫРЯНСКОМ КРАЮ [Dans le pays zyriène], С. КУРНИН И КО [S. Kurnin \& Co.], Mockba [Moscou].

ARSEN'EV Flegont Arsenevich АРСЕНЬЕВ ФЛЕГОНТ АРСЕНЬЕВИЧ, 1859, «ЗЫРЯНСКАЯ КОРРЕСПОНДЕНЦИЯ» [Correspondance zyriène], in LIMEROVA B. А. ЛИМЕРОвА В. A. (eds.), ЗЫРЯНЕ И ЗЫРЯНСКИЙ КРАЙ В ЛИТЕРАТУРНЫХ ДОКУМЕНТАХ ХІХ ВЕКА [Les Zyriènes et la terre zyriène dans les documents littéraires du XIXе siècle], ИЗДАТЕЛЬСТВО КОЛА [Éditions Kola], СЫкТЫВКАР [Syktyvkar], pp. 37-42.

ARSEN'EV Flegont Arsenevich АРсЕНЬЕВ ФЛЕГОНТ АРСЕНЬЕВИЧ, 1873, «ЗЫРЯНЕ И ИХ ОХОТНИЧЬИ ПРОМЫСЛЫ» [Les Zyriènes et la chasse], in LIMERovA В. А. лимЕРовА В. А. (eds.), зЫРЯНЕ И ЗЫРЯНСКИЙ КРАЙ В ЛИТЕРАТУРНЫХ ДОКУМЕНТАХ ХІХ ВЕКА [Les Zyriènes et la terre zyriène dans les documents littéraires du XIXе siècle], ИЗДАТЕЛЬСТВО КОЛА [Éditions Kola], СЫКТЫВКАР [Syktyvkar], pp. 37-42.

BALOV Evgenij Aleksandrovich БАЛОВ ЕВГЕНИЙ АЛЕКСАНДРОВИЧ, 1840, «ОХОТА ЗА БЕЛКОЙ И ДРУГИМИ ПУШНЫМИ ЗВЕРЯМИ В УСТЬСЫСОЛЬСКОМ, ЯРЕНСКОМ И СОЛЬВЫЧЕГОДСОМ УЕЗДАХ» [La chasse à l'écureuil et aux autres bêtes à fourrure dans les uezd d'Ust'-Sysol'sk, de Jarensk et de Sol'vyčegda], in LIMEROVA В. А. ЛИМЕРОВА В. А. (eds.), ЗЫРЯНЕ И ЗЫРЯНСКИЙ КРАЙ В ЛИТЕРАТУРНЫХ ДОКУМЕНТАХ ХІХ ВЕКА [Les Zyriènes et la terre zyriène dans les documents littéraires du XIXe siècle], изДАТЕЛЬСтво КОЛА [Éditions Kola], СЫктывКАР [Syktyvkar], pp. 13-17.

ВUNAKOV Nikolaj Fedorovich БУНАКОВ НИКОЛАЙ ФЕДОРОВИЧ, 1857, «ЗВЕРИНЫЙ ПРОМЫСЕЛ В ВОлОГОДСКОЙ гУБЕРНИИ» [La chasse aux bêtes sauvages dans le gouvernement de Vologda], in LIMEROVA B. А. ЛИМЕРОВA B. A. (eds.), ЗЫРЯНЕ И ЗЫРЯНСКИЙ КРАЙ В ЛИТЕРАТУРНЫХ ДОКУМЕНТАХ ХІХ $B E К A$ [Les Zyriènes et la terre zyriène dans les documents littéraires du XIXe siècle], ИЗДАТЕЛЬСТВо КОЛА [Éditions Kola], сыктывКАР [Syktyvkar], pp. 30-37.

DURKHEIM Emile, 1893, The Division of Labour in Society, The Free Press, New York, 462 p. FULLER Chris, 1994, "Legal Anthropology, Legal Pluralism and Legal Thought" in Anthropology Today, $\mathrm{n}^{\circ} 3$, vol. 10, pp. 9-12.

GEERTZ Clifford, 1983, Local Knowledge: Further Essays in Interpretative Anthropology, Basic Books, New York, 250 p.

IL'INA Irina, ИЛЬИНА ИРИНА ВАСИЛЬЕВНА \& ULJASHEV Oleg УЛЯШЕВ ОЛЕГ ИВАНОВИЧ, 2009, МУЖЧИНА И ЖЕНЩИНА В ТРАДИЦИОННОЙ КУЛЬТУРЕ КОМИ ВЕКА [L'homme et la femme dans la culture traditionnelle du siècle komi], ИНСТИТУТ ЯЗЫКА, ЛИТЕРАТУРЫ И ИСТОРИИ КОМИ НЦ УРО РАН [Institut de langue, littérature et histoire de Komi NC UrO RAN], сыктывКAР [Syktyvkar].

КONAKOV Nikolaj Dimitrievič КОНАКОВ НИКОЛАЙ ДМИТРИЕВИЧ, 1983, КОМИ ОХОТНИКИ И РЫБОЛОВЫ ВО ВТОРОЙ ПОЛОВИНЕ ХІХ - НАЧАЛЕ ХХ В. КУЛЬТУРА ПРОМЫСЛОВОГО НАСЕЛЕНИЯ ТАЕЖНОЙ ЗОНЫ ЕВРОПЕЙСКОГО СЕВЕРО ВОСТОКА [Les chasseurs et les pêcheurs komis dans la deuxième moitié du XIXe siècle et du début du XXe siècle. La culture de la population des la zone de taïga du nord-est européen], НАУКА [Science], Mockba [Moscou], 246 p.

LATKIN Vasilij, 1853, «ДНЕВНИК ВАСИЛИЯ НИКОЛАЕВИЧА ЛАТКИНА, ВО ВРЕМЯ ПУТЕШЕСТВИЯ НА ПЕЧОРУ, В 1840 И 1843 ГОДАХ, ЧАСТЬ ПЕРВАЯ» [Notes de la société géographique impériale russe, fascicule VII Zyriènes et la terre zyriène dans les documents littéraires du XIXe siècle] in ЗАПИскИ 
ИМПЕРАТОРСКОГО РУССКОГО ГЕОГРАФИЧЕСКОГО ОБЩЕСТВА, КНИЖКА VII ,[Journal de Vasilij Nikolaevič Latkin lors de son voyage sur la Pečora en 1840 et 1843, première partie] $n^{\circ} 7$. LEETE Art, 2011, « Komi jahimeestest ja koertest » in RUNNEL Pille, AGNES Adjas, KAALEP Tuuli \& SIKKA Toivo (eds.), Eesti Rahva Muuseumi aastaraamat, vol. 54, pp. 162-183.

LEETE Art \& LIPIN Vladimir, 2012, “Komi Hunter Narratives”, in BoWMAN Marion \& VALK Ülo (eds.), Vernacular Religion in Everyday Life: Expressions of Belief, Routledge, London, pp. 282-300.

LEETE Art \& LIPIN Vladimir, 2015, “The Concept of Truth in the Komi Hunting Stories" in Acta Borealia, vol. 32, pp. 68-84.

LIPIN Vladimir \& LEETE Art, 2000, "Komi Hunter Ethics at the End of the 20th Century" in Pro Ethnologia, vol. 10, pp. 77-85.

LIPIN Vladimir \& LEETE Art, 2015, « Lihtsad ja varjatud sõnumid tänaste komi küttide juttudes » in RUNNEL Pille, AGNES Adjas, KAALEP Tuuli \& KASTEHEIN Karin (eds.), Eesti Rahva Muuseumi aastaraamat, vol. 58, pp. 14-35.

MALINOWSKI Bronislaw, 1926, Crime and Custom in Savage Society, Harcourt, Brace \& Company ; Kegan Paul, Trench, Trubner \& Co., New York \& London, 132 p.

MIHAJLOV Mihajl БУНАКОВ НИКОЛАЙ ФЕДОРОВИЧ, 1851, «ПРОМЫСЛЫ ЗЫРЯН УСТЬСЫСОЛЬСКОГО И ЯРЕНСКОГО УЕЗДОВ ВОЛОГОДСКОЙ ГУБЕРНИИ» [La chasse et la pêche des Zyriènes des uezd d'Ust'-Sysol'sk et de Jarensk du gouvernement de Vologda], in LIMEROVA B. A. \& ЛИМЕРОВА В. A. (eds.), ЗЫРЯНЕ И ЗЫРЯНСКИЙ КРАЙ В ЛИТЕРАТУРНЫХ ДОКУМЕНТАХ ХІХ ВЕКА [LeS Zyriènes et la terre zyriène dans les documents littéraires du XIXе siècle], иЗДАТЕЛЬСТВО колА [Éditions Kola], сыктывкАР [Syktyvkar], pp. 21-23.

NALIMOV Vasilij Petrovich НАЛИМОВ ВАСИЛИЙ ПЕТРОВИЧ, 1924, «К ЭТНОЛОГИИ КОМИ (НАБРОСКИ)» [Sur l'ethnologie des Komis (esquisses)], in NALIMov Vasilij Petrovich НАЛИМОВ ВАСИЛИЙ ПЕТРОВИЧ (eds.), ОЧЕРКИ ПО ЭТНОГРАФИИ ФИННО УГОРСКИХ НАРОДОВ [Essais sur l'ethnographie des peuples finno-ougriens], МИНИСТЕРСТВО НАЦИОНАЛЬНОЙ ПОЛИТИКИ РЕСПУБЛИКИ КОМИ, УДМУРТСКИЙ ИНСТИТУТ ИСТОРИИ, ЯЗЫКА И ЛИТЕРАТУРЫ УРО РАН, ИНСТИТУТ ЯЗЫКА, ЛИТЕРАТУРЫ И ИСТОРИИ КОМИ НЦ УРО РАН [Ministère de la politique nationale de la République komie, Institut oudmourte d'histoire, de langue et de littérature UrO RAN, institut de langue et littérature du CN komi UrO RAN], ижЕВск \& СЫктЫВкAP [Iževsk \& Syktyvkar], pp. 75-84.

NALIMOV Vasilij Petrovich НАЛИМОВ ВАСИЛИЙ ПЕТРОВИЧ, 1926, «ОЧЕТ ЭТНОГРАФИЧЕСКИЙ эКСПЕДИЦИЙ ЗА 1926 Г» [Compte rendu de l'expédition ethnographique de 1926], in NALIMOV Vasilij Petrovich НАЛИМОВ ВАСИЛИЙ ПЕТРОВИЧ (eds.), ОЧЕРКИ ПО ЭТНОГРАФИИ ФИННО УГОРСКИХ НАРОДОВ [Essais sur l'ethnographie des peuples finno-ougriens], МИНИСТЕРСТВО НАЦИОНАЛЬНОЙ ПОЛИТИКИ РЕСПУБЛИКИ КОМИ, УДМУРТСКИЙ ИНСТИТУТ ИСТОРИИ, ЯЗЫКА И ЛИТЕРАТУРЫ УРО РАН, ИНСТИТУТ ЯЗЫКА, ЛИТЕРАТУРЫ И ИСТОРИИ КОМИ НЦ УРО РАН [Ministère de la politique nationale de la République komie, Institut oudmourte d'histoire, de langue et de littérature UrO RAN, institut de langue et littérature du CN komi UrO RAN], ижЕВсК \& сыктыВКАР [Iževsk \& Syktyvkar], pp. 174-318.

POPOv Klavdij попов КЛАВДИЙ, 1874, «ЗЫРЯНЕ И ЗЫРЯНСКИЙ КРАЙ» [Les Zyriènes et la terre zyriène] in ИЗВЕСТИЯ ИМПЕРАТОРСКОГО ОБЩЕСТВА ЛЮБИТЕЛЕЙ ЕСТЕСТВОЗНАНИЯ, АНТРОПОЛОГИИ И ЭТНОГРАФИИ, ${ }^{\circ} 2$ [Nouvelles de la société impériale des amateurs des sciences naturelles, d'anthropologie et d'ethnographie, Travaux de la section d'ethnographie], vol. 13. 
SIDOROv Aleksej Semenovich сидоРОВ АЛЕКСЕЙ СЕМЕНОВИЧ, 1928, ЗНАХАРСТВО, КОЛДОВСТВО И ПОРЧА У НАРОДА КОМИ. МАТЕРИАЛЫ ПО ПСИХОЛОГИИ КОЛДОВСТВА [Magie, sorcellerie et sorts chez les Komie], ИЗДАТЕЛЬСТВО «АЛЕТЕЙЯ» [Éditions « Aleteja »]., САНКТ ПЕТЕРБУРГ [Saint-Pétersbourg], $269 \mathrm{p}$.

SURVO Arno СУРBO APHO \& ŠARAPOV Valerij ШАРАПОВ ВАЛЕРИЙ, 2015, «“САМЫЙ ЖИЗНЕСПОСОБНЫЙ СРЕДИ НАРОДОВ ВОСТОЧОЙ РОССИИ" (ОБ ОДНОЙ НЕИЗВЕСТНОЙ ПУБЛИКАЦИИ В. П. НАЛИМОВА)» [Le plus vital parmi les peuples de la Russie orientale] in ВЕСТнИк УДМУРТСКОГО УНИВЕРСИТЕТА, $\mathrm{n}^{\circ} 1$ [Bulletin de l’Université oudmourte], vol. 25, pp. 162-169.

SVENSSON Tom G., 2002, "Indigenous Rights and Customary Law Discourse: Comparing the Nisga'a and the Sámi" in The Journal of Legal Pluralism and Unofficial Law, $\mathrm{n}^{\circ}$ 47, vol. 34, pp. 1-35, DOI : 10.1080/07329113.2002.10756562.

VOLKOV Nikolaj Dmitrievich волков НИКОЛАЙ ДМИТРИЕВИЧ, 1879, «УДОРСКИЙ КРАЙ. (ЭТНОГРАФИЧЕСКИЙ ОЧЕРК)» [La région de l'Udora (essai ethnographique)], in LIMEROVA B. A. ЛИМЕРОВА В. А. (eds.), ЗЫРЯНЕ И ЗЫРЯНСКИЙ КРАЙ В ЛИТЕРАТУРНЫХ ДОКУМЕНТАХ ХІХ ВЕКА [LeS Zyriènes et la terre zyriène dans les documents littéraires du XIXe siècle], ИзДАТЕЛЬСТВО колА [Éditions Kola], сыктывкар [Syktyvkar], pp. 53-68.

WIPO, 2013, "Customary Law, Traditional Knowledge and Intellectual Property: An Outline of the Issues", in World Intellectual Property Organization, Geneva, pp. 1-32, http://www.wipo.int/ export/sites/www/tk/en/resources/pdf/overview_customary_law.pdf.

ŽAKOv Kallistrat ЖАКОВ КАЛИСТРАТ, 1901, «ЭТНОЛОГИЧЕСКИЙ ОЧЕРК ЗЫРЯН» [Essai ethnologique des Zyriènes], in ŽAKOV Kallistrat ЖАКОВ КАЛИСТРАТ (eds.), ПОД ШУМ СЕВЕРНОГО ВЕТРА. РАССКАЗЫ, ОЧЕРКИ, СКАЗКИ И ПРЕДАНИЯ [Sous le bruit du vent du Nord. Récits, essais, contes et légendes], кОМИ КНИЖНОЕ ИЗДАТЕЛЬСтво [Éditions livres komis], СЫктЫВКАР [Syktyvkar], pp. 314-351.

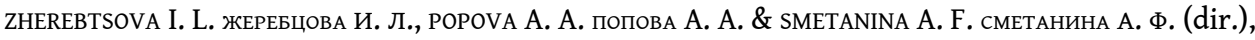
2011, ИСТОРИЯ КОМИ С ДРЕВНЕЙШИХ ВРЕМЕН ДО СОВРЕМЕННОСТИ Istorija komi [L'histoire de Komi des temps ancestraux jusqu'à nos jours], vol. 1, «АНБУР» [« Anbur »], СЫКТЫВКАР [Syktyvkar], $544 \mathrm{p}$.

\section{Sources}

TT 1996-2016 = matériaux de terrain des auteurs, 1996-2016

\section{NOTES}

1. Cet article a été mis au point avec le soutien de la subvention PUT590 de l'Agence estonienne pour la recherche (Eesti Teadusagentuur) du projet $\mathrm{nr} 782$ du programme estonien des Peuples finno-ougriens.

2. Dans cet article, nous indiquons systématiquement l'année de collecte, l'âge de l'informateur et nous faisons appel à des pseudonymes, pour garanti l'anonymat des personnes.

3. Pokrov - journée dans le calendrier orthodoxe russe dédiée à la protection et à l'intercession de la Vierge, le 14 octobre. 


\section{RÉSUMÉS}

Dans cet article, nous étudions le droit coutumier des chasseurs komis aujourd'hui, tout en le replaçant dans son contexte historique. Nous commençons par décrire les principes et les règles $\mathrm{du}$ droit coutumier, puis nous montrons comment les coupables sont identifiés et finalement nous analysons les mesures punitives que les chasseurs komis mettent en œuvre. Nous trouvons que le droit coutumier des chasseurs komis se distingue nettement du système juridique de l'État, même s'il a connu des points de rencontre avec le droit officiel au cours des temps. Le droit coutumier komi repose sur une vision du monde animiste, bien qu'aujourd'hui les chasseurs eux-mêmes ne mettent pas l'accent sur cette dimension.

Käesoleva artikliga uurime komi jahimeeste tänapäevast tavaõigust, seades selle samas ka ajaloolisse konteksti. Kõigepealt kirjeldame komi tavaõiguse põhimõtteid ja reegleid, seejärel esitame andmed viisidest, kuidas tavaõiguse rikkujaid tabatakse ja lõpuks analüüsime komi küttide poolt rakendatavaid karistusmeetmeid. Leiame, et komi jahimeeste tavaõigus on selgelt eristunud riiklikust õigussüsteemist, kuigi sellel on ametliku õigusega eri aegadel olnud ka erineval määral kokkupuuteid. Komi tavaõigus põhineb animistlikul maailmapildil, olgugi, et tänapäeval kütid seda seost väga tihti ei rõhuta.

\section{INDEX}

Index chronologique : XXIe siècle début

Mots-clés : oudmourte, komis, animisme, droit coutumier

Thèmes : anthropologie, anthropologie juridique

Keywords : Udmurt, 21th century beginning, anthropology, komis

nomsmotscles Komis, Mansis, Nénetses, Ougriens, Ougriens de l’Ob, Russes

Index géographique : Oural, Russie, Syktyvkar 